\title{
DIFFERENTIAL EQUATIONS WITH GENERAL BOUNDARY CONDITIONS
}

\author{
WILLIAM M. WHYBURN
}

This paper concerns ordinary differential equations in the real domain. More specifically, it discusses systems of first order, nonsingular, equations of the form

$$
\frac{d y_{i}}{d x}=f_{i}\left(x, y_{1}, y_{2}, \cdots, y_{n}\right), \quad i=1,2, \cdots, n,
$$

together with boundary conditions at one, two, or more points of the interval of definition. System (1) is quite general and can be made to include the nonsingular equations of the $n$th order as special cases. The paper makes no attempt to generalize the equations beyond those that occur in standard treatments. Any elements of generality or novelty introduced appear in connection with the types of boundary conditions discussed. The mathematical literature of the past century contains many results for system (1) with boundary conditions at one point - the so-called fundamental existence theorems occupying central positions-and with boundary conditions at two points of the interval where second order linear systems have been of prime importance. These important bodies of results have been suveyed before the Society through addresses by Bôcher, Bliss, Pell-Wheeler, Reid and others, and therefore are given little mention in the present paper. We discuss results that have been obtained in cases where the boundary conditions apply to $n$ or less points, to any finite number of points, to any infinite point set of the first species, ${ }^{1}$ and where the boundary conditions contain integrals over an interval. The literature on differential systems with such boundary conditions is not extensive although many substantial results have been obtained and potential applications exist in a number of fields.

1. Fundamental existence theorems. Let the real functions $f_{i}\left(x, y_{1}, \cdots, y_{n}\right)$ in system (1) be defined over a domain

$$
D: \quad\left\{\begin{array}{l}
(a, b): a \leqq x \leqq b \\
R: A_{i}<y_{i}<B_{i} \quad(i=1, \cdots, n),
\end{array}\right.
$$

An address delivered before the Pasadena meeting of the Society on November 22, 1941, by invitation of the Program Committee; received by the editors May 28, 1942.

1 A set of the first species is one which has at most a finite number of nonvacuous derived sets. Any such set is at most denumerably infinite. See Hobson, Theory of Functions of a Real Variable, vol. 1, 2d edition, Cambridge, 1920, pp. 71 and 79. 
of $(n+1)$-dimensional real space, where $a$ and $b$ are finite but any or all of the $A_{i}$ 's and $B_{i}$ 's may be infinite. By a solution of system (1) on an interval $\alpha \leqq x \leqq \beta$ we understand a set of absolutely continuous functions $y_{i}=g_{i}(x)(i=1,2, \cdots, n)$, such that the equations $g_{i}^{\prime}=f_{i}\left[x, g_{1}(x), g_{2}(x), \cdots, g_{n}(x)\right](i=1,2, \cdots, n)$, hold at all points of $\alpha \leqq x \leqq \beta$ except possibly for a set of points of measure zero. Classical fundamental existence theorems for this system are of "neighborhood" and "interval" types. The first of these uses light hypotheses but gives existence of solutions in neighborhoods of specified points only. The second or "interval" type uses strong hypotheses and yields existence throughout previously assigned intervals. A typical theorem of the first type follows:

THEOREM I. Let the functions $f_{i}\left(x, y_{1}, \cdots, y_{n}\right)$ be continuous in $\left(y_{1}, \cdots, y_{n}\right)$ throughout $R$ for each fixed $x$ on $(a, b)$, and measurable in $x$ on $(a, b)$ for fixed $\left(y_{1}, \cdots, y_{n}\right)$ in $R$. Let a Lebesgue integrable function $M(x)$ exist on $(a, b)$ such that throughout $D,\left|f_{i}\left(x, y_{1}, \cdots, y_{n}\right)\right|<M(x)$ $(i=1,2, \cdots, n)$. If $P\left(c, k_{1}, k_{2}, \cdots, k_{n}\right)$ is any point of $D$, there exists a neighborhood of $x=c$ on which equation (1) has at least one solution such that $y_{i}(c)=k_{i}(i=1,2, \cdots, n)$.

Theorem $I$ is due to Caratheodory ${ }^{2}$ and contains the Peano type theorems where the functions $f_{i}\left(x, y_{1}, \cdots, y_{n}\right)$ are assumed continuous throughout $D$, as special cases. For the latter theorems, the uniform boundedness condition is a consequence of the assumed continuity. If the domain $D$ is such that $A_{1}, \cdots, A_{n}$, are negatively infinite while $B_{1}, \cdots, B_{n}$ are positively infinite, then Theorem I becomes the following "interval" type theorem:

THEOREM II. Let the functions $f_{i}\left(x, y_{1}, \cdots, y_{n}\right)$ be continuous in $\left(y_{1}, \cdots, y_{n}\right)$ for each fixed $x$ on $(a, b)$ and all real $\left(y_{1}, \cdots, y_{n}\right)$ and let these functions be measurable in $x$ on $(a, b)$ for any fixed $\left(y_{1}, \cdots, y_{n}\right)$ and, finally, let a Lebesgue integrable function $M(x)$ exist such that

$$
\left|f_{i}\left(x, y_{1}, \cdots, y_{n}\right)\right|<M(x), \quad i=1, \cdots, n,
$$

for all $x$ on $(a, b)$ and all real $\left(y_{1}, \cdots, y_{n}\right)$. If $x=c$ is a point of $(a, b)$ and $k_{1}, \cdots, k_{n}$ are real numbers, there exists at least one solution of system $(1)$ on $(a, b)$ such that $y_{i}(c)=k_{i}(i=1,2, \cdots, n)$.

Through use of over and under functions, Perron and others have obtained "interval" type existence theorems ${ }^{3}$ which differ materially

2 Vorlesungen über reelle Funktionen, Leipzig, 1928, p. 672.

${ }^{3}$ For discussions of these theorems, see W. M. Whyburn, University of California Publications in Mathematical and Physical Sciences, vol. 1 (1935), pp. 115-134. 
in hypotheses from those stated above and which frequently apply when Theorems I and II fail.

The hypotheses of Theorem II exclude linear systems. Separate existence theorems may be proved for such systems or Theorem II may be modified in such a way that it includes them as special cases. The latter result ${ }^{4}$ is obtained by replacing $f_{i}\left(x, y_{1}, \cdots, y_{n}\right)$ with $F_{i}\left(x, y_{1}, \cdots, y_{n}\right)+\sum_{j=1}^{n} G_{i j}\left(x, y_{1}, \cdots, y_{n}\right) y_{j}$ and requiring that $F_{i}$, $G_{i j}$, satisfy the same hypotheses originally stated for $f_{i}\left(x, y_{1}, \cdots, y_{n}\right)$.

Niccoletti ${ }^{5}$ studied a single differential equation of the $n$th order together with initial conditions at more than one point of the interval. The actual number of such points did not exceed $n$, the order of the differential equation. Since this work seems to have been the first on differential systems with boundary conditions at more than two points, we indicate the results obtained and the nature of the methods used. Through the use of certain algebraic formulas, Niccoletti established the fact that the function

$u=\frac{1}{(n-1) !} f(x) \sum_{i=1}^{k} \frac{\partial^{\alpha_{i}-1}}{\partial a_{1 i}^{\alpha_{i}-1}}\left\{\frac{\alpha_{i}}{f^{\left(\alpha_{i}\right)}\left(a_{i}\right)\left(x-a_{i}\right)} \int_{a_{i}}^{x}\left(a_{i}-z\right)^{n-1} \phi(z) d z\right\}$,

where $f(x)=\left(x-a_{1}\right)^{\alpha_{1}}\left(x-a_{2}\right)^{\alpha_{2}} \cdots\left(x-a_{k}\right)^{\alpha_{k}}, \alpha_{1}+\alpha_{2}+\cdots+\alpha_{k}=n$, vanishes along with its first $\left(\alpha_{i}-1\right)$ derivatives at the point $x=a_{i}$ $(i=1,2, \cdots, k)$. Furthermore $u^{(n)}=\phi(x)$. If $P_{n-1}(x)$ is a polynomial of degree $n-1$ which together with its first $\alpha_{i}-1$ derivatives takes assigned values at $x=a_{i}(i=1,2, \cdots, k)$, then $y(x)=u(x)+P_{n-1}(x)$ is a function which satisfies the differential equation $y^{(n)}=\phi(x)$ together with boundary conditions which assign the values of $y(x)$ and its first $\alpha_{i}-1$ derivatives at the points $x=a_{i}(i=1,2, \cdots, k)$. With this result and the Picard method of successive approximations, Niccoletti proved the following theorem:

THEOREM III. In the differential equation

$$
y^{(n)}=\phi\left(x, y, y^{\prime}, \cdots, y^{(n-1)}\right)
$$

let the function $\phi\left(x, y, y^{\prime}, \cdots, y^{(n-1)}\right)$ be continuous for all $x$ on $(a, b)$ and all real $\left(y, y^{\prime}, \cdots, y^{(n-1)}\right)$ and let it satisfy a Lipschitz condition with respect to $\left(y, y^{\prime}, \cdots, y^{(n-1)}\right)$, then there exists a unique solution of (2) which together with its first $\alpha_{i}$ derivatives takes on assigned values at $x=a_{i}(i=1, \cdots, k)$, provided the points $a_{1}, a_{2}, \cdots, a_{k}$ all lie on $a$ single subinterval of $(a, b)$ that is sufficiently small.

${ }^{4}$ See W. M. Whyburn, Annals of Mathematics, (2), vol. 30 (1928), pp. 31-38.

${ }^{5}$ Atti della R. Accademie Delle Scienze Di Torino, vol. 33 (1897), pp. 746 ff. 
Niccoletti indicated that his results could be extended to systems of differential equations and to partial differential equations, but it seems clear that the actual extension to systems was not accomplished. Otherwise, the restriction that $y, y^{\prime}, \ldots, y^{(\alpha-1)}$ be assigned at any point where $y^{(\alpha)}$ is given would have been unnecessary. Niccoletti stated that his solutions were unique and that they were continuous functions of the initial values.

Independently of the work of Niccoletti, W. M. Whyburn ${ }^{6}$ established a theorem of the type of Theorem III for systems (1) with initial conditions at $n$ or less points of the interval. This theorem requires that the points lie in a single subinterval of $(a, b)$ of length equal to a definite positive number but establishes existence and uniqueness of the solution on the entire interval $(a, b)$. The solution functions are continuous functions of the initial points and values. The essential character of the requirement that the points used in the initial conditions be contained in a restricted subinterval of $(a, b)$ is shown by the example $y^{\prime}=k z, z^{\prime}=-k y, y\left(a_{1}\right)=\alpha, z\left(a_{2}\right)=\beta, \alpha^{2}+\beta^{2} \neq 0$. A solution exists here if $\left|a_{1}-a_{2}\right|<\pi / 2 k$ but fails to exist when $a_{2}-a_{1}=\pi / 2 k$.

Theorems of the above type are important since they allow the study of differential systems under conditions which do not permit the simultaneous assignment of all of the initial values but which do admit this assignment over a relatively short range of the independent variable. One possible application would be to a study of rapid machine gun fire where the gun is moved about during the firing interval and the individual trajectories are not as important as the family of such paths generated over a relatively short interval of time. Here one would have a system of second order equations-in number equal to the number of times the gun is fired-together with $2 n$ initial conditions assigned at the times $t_{1}, t_{2}, \cdots, t_{n}$, when the gun actually fires. The above theorems, together with approximation methods which grow out of them, would apply to such problems.

This section of the paper is closed with a brief consideration of linear systems in which the notation used in the remainder of the paper is indicated. With the adaptation mentioned above, fundamental existence theorems apply to a system of first order linear differential equations with coefficients which are Lebesgue integrable functions of $x$ on $(a, b)$. It is convenient to write these systems in matrix form ${ }^{7}$

${ }^{6}$ Annals of Mathematics, (2), vol. 30 (1928), pp. 31-38.

7 This notation has been used by many writers and is becoming quite standard in the literature. One of many references to its use is Birkhoff and Langer, Proceedings of the American Academy of Arts and Sciences, vol. 57 (1922), pp. 51-128. 


$$
L(Y)=Y^{\prime}+P(x) Y=Q(x),
$$

where $L(Y), P, Y, Q$, and $Y^{\prime}$ are square matrices of $n$-rows. If $x=s$ is a point of $(a, b)$ and if initial conditions are given at this point, the method of variation of parameters yields

$$
Y(x)=U(x)\left[\int_{s}^{x} V(t) Q(t) d t+V(s) Y(s)\right],
$$

where $U(x)$ is a nonsingular matrix solution of the homogeneous equation $L(Y)=0$ and $V(t)=U^{I}(t)$ is the inverse of $U(t)$. This formula is useful in the remainder of the paper and is important in its own right since it is an explicit expression for the general solution of equation (3) in terms of a solution of the homogeneous equation and the initial values at one point. It is especially useful in a study of the solution of (3) as a functional of the coefficients and initial values where it provides estimates of the effects on the solution produced by small variations in the coefficients of the system. ${ }^{8}$

2. Boundary conditions at two points. Extensive investigations have been made of system (1) with conditions at two points of the interval. By far the greater part of these studies have been devoted to second order linear systems with parameters in the coefficients. The present paper omits ${ }^{9}$ discussion of these results for second order systems and mentions only one development for $n$th order linear systems-namely that of the Green's function ${ }^{10}$ or matrix-which is of interest in connection with later parts of the paper.

Let system (3) be given along with boundary conditions

$$
H(Y)=A Y(a)+B Y(b)=C
$$

where $A, B, C$ are $n$th order square matrices of constants. If the homogeneous system $L(Y)=0, H(Y)=0$, is incompatible, then the existence and properties of the Green's matrix may be deduced in the following manner. In formula (4) let $s=a$ and multiply the resulting equation on the left by $A U(a) V(x)$. Similarly, replace $s$ in formula (4) by $b$ and multiply the resulting equation on the left by $B U(b) V(x)$. Addition of these two equations followed by multiplication on the left by $U(x) H^{I}(U)$ yields

$$
Y(x)=U(x) H^{I}(U) C+\int_{a}^{b} G(x, t) Q(t) d t,
$$

${ }^{8}$ See W. M. Whyburn, Transactions of this Society, vol. 32 (1930), pp. 502-508.

${ }^{9}$ For references on this literature, see Reid, this Bulletin, vol. 43 (1937), pp. 633666.

${ }^{10}$ See Bounitsky, Journal de Mathématiques, vol. 5 (1909), pp. 65-125. 
where

$$
G(x, t)=\left\{\begin{aligned}
U(x) H^{I}(U) A U(a) V(t) & \text { for } t<x \\
-U(x) H^{I}(U) B U(b) V(t) & \text { for } \quad t>x .
\end{aligned}\right.
$$

$G(x, t)$ as given by (7) is the Green's matrix for the system and its properties of uniqueness, continuity, symmetry, and so on, are readily deduced from this explicit formula.

Our discussion of two point boundary conditions is concluded with the observation that, in general, the conditions apply to the end points of the interval under consideration and hence at the boundary of the simply-connected domain (interval) of definition. Cases where one or both of the points are interior to the interval are usually treated by studying the solution on the interval bounded by these points and extending this work to the larger interval through use of results for boundary conditions at one point.

3. Conditions at a finite number of points. In this section we consider $n$th order linear systems with boundary conditions which involve a finite number $k$ of points of the interval. For the differential system (3) the boundary conditions have the form

$$
H(Y)=\sum_{j=1}^{k} A_{j} Y\left(a_{j}\right)=C,
$$

where $A_{1}, \cdots, A_{k}, C$, are constant matrices and $a_{1}<a_{2}<\cdots<a_{k}$ are $k$ distinct points of $(a, b)$. For the single linear differential equation of the $n$th order, the boundary conditions are linear combinations of the values of the solution and its first $(n-1)$ derivatives at the $k$ points. M. Picone ${ }^{11}$ seems to have been the first person to formulate boundary conditions of type. He also obtained some results for a special $n$th order differential equation with integral boundary conditions. Bôcher discussed such conditions in his Fifth International Congress address $^{12}$ of 1912 and in his Paris lectures ${ }^{13}$ of 1913-1914. Bôcher indicated the importance of boundary value problems of this type but did not publish any investigations on them. The first systematic study of such problems was made by C. E. Wilder, ${ }^{14}$ who treated the single $n$th order equation with conditions at $k$ points. Wilder defined a

11 Rendiconti della Accademia dei Lincei, vol. 17 (1908), pp. 340-347.

12 Proceedings of the Fifth International Congress of Mathematicians, Cambridge, 1912, vol. 1, pp. 163-195.

${ }^{13}$ Bôcher, Lȩ̧ons sur les Methodes de Sturm, Paris, 1917, p. 18.

${ }_{14}$ Transactions of this Society, vol. 18 (1917), pp. 415-442, and vol. 19 (1918), pp. $157-166$. 
Green's function for his differential system and investigated the adjoint relationship. He then introduced a parameter into one of the coefficients of the equation and developed expansion theorems for the resulting system making use of the Green's function and methods first introduced by Birkhoff. ${ }^{15}$ Existence and oscillation theorems for second order systems containing a parameter in a general fashion and with boundary conditions, one of which involved $k$ points, were obtained by W. M. Whyburn. ${ }^{16}$ The results here differed from those ordinarily obtained for two point conditions in that the characteristic numbers were not isolated but occurred in sets or in bands. This, in itself, is of interest since the occurrence of bands of characteristic numbers in spectral analysis is frequent. Toyada ${ }^{17}$ studied the Green's function and adjoint relationship for $n$th order differential systems, apparently overlooking the earlier work of Wilder. His results are quite similar to those obtained by Wilder although his method of attack differed in that he did not raise the dimensionality of the space or increase the order of the differential system. Toyada used the skewsymmetry of the Green's matrix as a basis for building an adjoint system and arrived at such a system under the condition that a total of $k n^{2}$ identities hold. He attempted to generalize his results to systems with integral boundary conditions but seems to have overlooked difficulties that arise due to the above-mentioned identities. Wilder's previous results showed that, in general, an adjoint system in the ordinary sense would not exist. This result has recently been confirmed by Mansfield. ${ }^{18}$

The connections between $k$-point boundary value problems and the calculus of variations have been explored by Reid, ${ }^{19}$ Denbow, ${ }^{20}$ Smiley ${ }^{21}$ and Mansfield. ${ }^{18}$ This work starts from the relation between the Jacobi necessary condition on the one hand, and second order differential equations with boundary conditions at two points on the other hand, and proceeds to cases where more than two points appear in the boundary conditions. An important device used is a transformation due to Reid ${ }^{19}$ which effectively reduces the $k$-point case to

${ }_{15}$ Transactions of this Society, vol. 9 (1908), pp. 373-395.

${ }_{16}$ Transactions of the Society, vol. 30 (1928), pp. 630-640.

${ }_{17}$ Tohôku Mathematical Journal, vol. 38 (1933), pp. 343-355, and vol. 39 (1934), pp. 387-398.

${ }^{18}$ Contributions to the Calculus of Variations, vol. 4, University of Chicago Press, pp. 413-472.

${ }^{19}$ American Journal of Mathematics, vol. 57 (1935), pp. 69-93.

${ }^{20}$ Contributions to the Calculus of Variations, 1933-1937, Chicago, 1937, pp. 449494.

${ }^{21}$ Ibid., pp. 527-566 
one that involves $k-1$ systems each of the two point type. Mansfield ${ }^{18}$ made a systematic study of $k$-point boundary value problems through use of this device and applied his results to the calculus of variations and to differential systems in the complex domain.

Much of the work mentioned above makes specific use of the fact that the number of points appearing in the boundary conditions is finite. In some cases, use is made of an increase in the dimensionality of the space while in other cases, the order of the differential system is raised. When it is observed that the introduction of interior points of the interval into the boundary conditions has the effect of changing the simply-connected domain of definition into one that is multiplyconnected, it is not surprising that devices of the above-mentioned type are used. However, difficulties arise when attempts are made to generalize the differential system through having the boundary conditions contain integrals or infinitely many points. The next two sections discuss conditions of these types.

4. Integral conditions. When the number of points which enter the boundary conditions is no longer required to be finite, there are possibilities that the conditions will contain linear combinations of the solution values on a non-dense infinite point set or contain definite integrals of the solution over the interval. Picone ${ }^{22}$ investigated relationships that exist between integral equations and certain special $n$th order differential equations with integral boundary conditions. von Mises ${ }^{23}$ studied the special second order system

$$
\left\{\begin{array}{l}
\frac{d}{d x}\left[k \frac{d u}{d x}\right]+(\lambda g-q) u=0 \\
\int_{a}^{b} A(x) u(x) d x=0, \quad \int_{a}^{b} B(x) u(x) d x=0,
\end{array}\right.
$$

where $k, g, q, A, B$ are continuous functions of $x$ and $\lambda$ is a parameter. von Mises was interested in this system because of its occurrence in certain problems of hydrodynamics. ${ }^{24}$ His special case of hydrodynamic application had $k=q=1, g=x+c, A=1 / B=e^{x}$, where $c$ is a constant. With specializations in the direction of this application, von Mises used Sturm's method of passage to the limit from an algebraic system to obtain existence and oscillation theorems for system (9).

\footnotetext{
${ }_{22}$ Loc. cit., pp. 340-347.

${ }^{23}$ Festschrift Heinrich Weber, Leipzig, 1912, pp. 252-282.

${ }^{24}$ See Sommerfeld, Proceedings of the Fourth International Congress of Mathematicians, Rome, 1909, vol. 3, p. 116.
} 
Bôcher ${ }^{25}$ mentioned integral boundary conditions for second order systems and indicated a transformation that could sometimes be applied to replace such conditions by conditions at two points. The transformation suggested by Bôcher depends upon the actual solution in closed form of a slightly modified form of the given differential equation and, for this reason, offers little probability of use in any particular problem. Bôcher observed that this transformation would replace system (9) by one which no longer involved the parameter in the simple linear fashion of its original occurrence. In general, systems with integral boundary conditions should be regarded as essentially different from those with two point or $k$-point conditions. It is clear that integral boundary conditions would arise quite naturally in hydrodynamics or indeed in any investigation where such things as fixed areas under solution curves, fixed lengths of curves, and so on enter.

Tamarkin ${ }^{26}$ considered $k$-point and integral conditions for single $n$th order linear differential equations.

W. M. Whyburn ${ }^{27}$ established existence and oscillation theorems for linear second order equations with one integral boundary condition and one condition of the two point type. The coefficients of the system contained a parameter in a general (non-linear) fashion. As in the case of $k$-point conditions, it was found that the characteristic numbers were not isolated but occurred in sets which might be continua. Toyada ${ }^{28}$ set up a Green's matrix for $n$th order linear systems with integral boundary conditions and attempted to generalize the adjoint notion to systems of this type. His methods were extensions of ones that he had used for $k$-point conditions and such extensions were possible because these methods were largely independent of the number of points involved in the boundary conditions. In connection with his work on adjoint systems, Toyada imposed a set of conditions the number of which depended upon the number of points involved in the boundary conditions. These conditions become unruly when integral conditions are used.

Integral boundary conditions of a different type have been investigated by a number of people. ${ }^{29}$ These conditions contain Stieltjes integrals and for the $n$th order linear system (3) have the form

${ }^{25}$ Proceedings of the Fifth International Congress of Mathematicians, Cambridge, 1912, vol. 1, p. 167.

${ }^{26}$ On Some General Problems of the Theory, (in Russian), Petrograd, 1917.

${ }^{27}$ Transactions of this Society, vol. 30 (1928), pp. 630-640.

${ }^{28}$ Loc. cit., p. 391.

${ }^{29}$ See N. Cioranescu, Bulletinul, Facultătea de Stiinte din Cernăuti, vol. 5 (1931), p. 99 and Mathematische Zeitschrift, vol. 35 (1932), pp. 601-608. Also see A. Smorgorshewsky, Recueil Mathématique, (n.s.), vol. 7 (1940), pp. 179-196. 


$$
H(Y)=\int_{a}^{b} d F(x) Y(x)=C,
$$

where $H(Y), C, F(x), Y$ are square matrices of $n$-rows and the elements of $F(x)$ are of bounded variation on $(a, b)$. Boundary conditions of this type are quite general and contain all of the conditions discussed above as special cases. For example, conditions (8) involving $k$ points of the interval are obtained when $F(x)$ is defined as a matrix of step functions-constant on each of the subintervals $a_{i}<x<a_{i+1}$ but with discontinuities at the points $x=a_{i}$ given by $F\left(a_{i}^{+}\right)-F\left(a_{i}\right)$ $=A_{i}(i=1, \cdots, n)$, where $F\left(a_{1}^{-}\right)=F(a), F\left(a_{k}^{+}\right)=F(b)$. In this case

$$
\int_{a}^{b} d F(x) Y(x)=\sum_{i=1}^{k} A_{i} Y\left(a_{i}\right)
$$

Integral boundary conditions of the type $\int_{a}^{b} F(x) Y(x) d x=C$ are reduced to the above type when use is made of the relations between Stieltjes integrals and those of Riemann or Lebesgue types. Smorgorshewsky ${ }^{30}$ developed the notions of Green's matrix and generalized Green's matrix for systems of type (3), (10), and investigated the properties of these matrices. The fact that linear boundary conditions of quite general type can be replaced by conditions containing Stieltjes integrals has been known for many years. However, partly due to the somewhat unwieldly character of Stieltjes integrals, few advantages have resulted from such replacement. The above-mentioned results for the Green's matrix represent one place, at least, where advances have been made through use of Stieltjes integrals.

5. Boundary conditions at infinitely many points. In this section $n$th order differential systems with boundary conditions at infinitely many points of the interval are discussed. The most general sets considered are those of the first species. If $g$ is a point set of the first species and if $A_{1}, A_{2}, \cdots$ is an absolutely convergent ${ }^{31}$ sequence of constant matrices, the boundary conditions for system (3) are of the form

$$
H_{1}(Y)=\sum_{1}^{\infty} A_{i} Y\left(d_{i}\right)=C_{1},
$$

where $d_{1}, d_{2}, \ldots$ is some ordering of the elements of $g$ into a countable sequence. It is a consequence of the absolute convergence of $\sum_{1}^{\infty} A_{i}$ that conditions (11) are equivalent to those obtained by any

${ }^{30}$ Loc. cit., pp. 179-196.

${ }^{31}$ That is to say, each element sequence converges absolutely. 
other countable ordering of the elements of $g$. More general boundary conditions are obtained if an integral term occurs in (11) as follows:

$$
H_{2}(Y)=\sum_{1}^{\infty} A_{i} Y\left(d_{i}\right)+\int_{a}^{b} F_{2}(x) Y(x) d x=C_{2} .
$$

In a forthcoming article ${ }^{32}$ the present author has shown that differential system (3) together with boundary conditions (12) is equivalent to a system consisting of (3) and conditions

$$
H(Y)=A Y(a)+B Y(b)+\int_{a}^{b} F(x) Y(x) d x=C .
$$

These systems are equivalent in the sense that any solution of the one system is also a solution of the other. Details of the proof are omitted here since they appear in the article cited. It is to be noted that the matrix function $F(x)$ is Lebesgue integrable on $(a, b)$ provided $F_{2}(x)$ has this property. In general, $F(x)$ is not continuous and may fail to be Riemann integrable. A further formal reduction of boundary conditions (13) is accomplished if $F(x)$ is assumed to have properties that permit replacement of the integral and two point terms by a single Stieltjes integral. However, this replacement does not actually simplify the system and does lose certain advantages that result from the form of conditions (13). To illustrate these advantages, we develop the notion of Green's matrix for system (3), (13) and thus for system (3), (12).

Let $U(x)$ and $V(x)$ have the same meanings as in formula (4) and let $Y(x)$ denote the general solution of equation (3). Multiplication of equation (4) on the left by $F(s) U(s) V(x) d s$ followed by integration, once between the limits $s=a$ and $s=x$ and once between the limits $s=b$ and $s=x$ yields

$$
\begin{aligned}
& \int_{a}^{x} F(s) U(s) V(x) Y(x) d s \\
& =\int_{a}^{x} F(s) Y(s) d s+\int_{a}^{x} d s \int_{a}^{x} F(s) U(s) V(t) Q(t) d t, \\
& \int_{b}^{x} F(s) U(s) V(x) Y(x) d s \\
& =\int_{b}^{x} F(s) Y(s) d s+\int_{b}^{x} d s \int_{s}^{x} F(s) U(s) V(t) Q(t) d t .
\end{aligned}
$$

${ }^{32}$ Soon to appear in University of California Publications in Mathematics, University of California Press, Los Angeles. 
When the double integrals in (14) and (15) are integrated by parts and the function

$$
G_{2}(x, t)= \begin{cases}\int_{a}^{t} F(s) U(s) V(t) d s & \text { for } t<x \\ \int_{b}^{t} F(s) U(s) V(t) d s & \text { for } t>x\end{cases}
$$

is introduced, equation (14) and (15) combine to yield

$$
\int_{a}^{b} F(t) U(t) V(x) Y(x) d t=\int_{a}^{b} F(t) Y(t) d t+\int_{a}^{b} G_{2}(x, t) Q(t) d t .
$$

Let $s=a$ in equation (4) and multiply this equation on the left by $A U(a) V(x)$. Similarly, let $s=b$ in equation (4) and multiply this equation on the left by $B U(b) V(x)$. Addition of the two resulting equations to equation (16) yields

$$
H(U) V(x) Y(x)=H(Y)+\int_{a}^{b}\left[G_{1}(x, t)+G_{2}(x, t)\right] Q(t) d t
$$

where

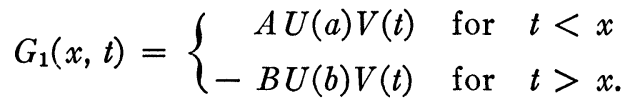

If the homogeneous system $L(U)=0, H(U)=0$ is assumed incompatible, then $H(U)^{I}$ exists and equation (17) yields the unique solution of system (3), (13) in the form

$$
Y(x)=U(x) H(U)^{I} C+\int_{a}^{b} G(x, t) Q(t) d t,
$$

where the Green's matrix $G(x, t)$ is given explicitly by

$$
G(x, t)=\left\{\begin{array}{r}
U(x) H(U)^{I}\left[A U(a)+\int_{a}^{t} F(s) U(s) d s\right] V(t) \text { for } t<x \\
-U(x) H(U)^{I}\left[B U(b)+\int_{t}^{b} F(s) U(s) d s\right] V(t) \text { for } t>x
\end{array}\right.
$$

$G(x, t)$ as defined by equation (19) has the usual properties of continuity, uniqueness, and so on, of the Green's matrix for systems with less general boundary conditions. If the function $F(x)$ is taken as the zero matrix, the differential system (3), (13) becomes the two point system (3), (5) and formula (19) shows immediately that $G(x, t)$ be- 
comes the Green's matrix defined by equation (7). Examination of formula (19) reveals the fact that symmetry of $G(x, t)$ in $x$ and $t$ is to be expected only in the degenerate two point case $F(x) \equiv 0$.

Although an adjoint system for (3), (13) in the ordinary sense does not exist in general, it is possible to set up boundary conditions similar to those given in (13) which can be taken with the adjoint equation

$$
M(Z)=Z^{\prime}-Z(x) P(x)=0
$$

to form a very useful system associated with (3), (13). When $F(x)$ $\equiv 0$ this system becomes the adjoint system for (3), (13).

Simultaneous study of the Green's matrices for (3), (13) and this associated system shows that they satisfy relations which readily degenerate into the well known symmetry relations for the case of two point systems.

6. Conclusion. This paper has attempted to review a body of literature on boundary value problems for ordinary differential equations which has not, to our knowledge, been summarized before. Although many of the results cited have appeared in print, it is believed that the paper is not without some novelty. It is hoped that presentation of the paper at this time will lead to applications of systems with more general boundary conditions in a number of fields of applied mathematics. The author does not believe that known results for such systems are adequate to handle many of these applications, but he does think that additional results can be obtained as needs for them arise in applied problems. It is also likely that many problems now treated as two point boundary value problems would receive better treatment if they were studied through the use of more general boundary conditions.

University of California at los Angeles 